\title{
DETECTION AND CLASSIFICATION OF POWER QUALITY DISTURBANCE WAVEFORM USING MRA BASED MODIFIED WAVELET TRANSFROM AND NEURAL NETWORKS
}

\author{
Perumal Chandrasekar — Vijayarajan Kamaraj *
}

\begin{abstract}
In this paper, the modified wavelet based artificial neural network (ANN) is implemented and tested for power signal disturbances. The power signal is decomposed by using modified wavelet transform and the classification is carried by using ANN. Discrete modified wavelet transforms based signal decomposition technique is integrated with the back propagation artificial neural network model is proposed. Varieties of power quality events including voltage sag, swell, momentary interruption, harmonics, transient oscillation and voltage fluctuation are used to test the performance of the proposed approach. The simulation is carried out by using MATLAB software. The simulation results show that the proposed scheme offers superior detection and classification compared to the conventional approaches.
\end{abstract}

K e y w or ds: modified wavelet transforms, power quality disturbances, AM, detection and classification, ANN

\section{INTRODUCTION}

The quality of electric power has become an important issue because, with the introduction and wide spread use of sensitive electronic equipment, customers have become much more aware and sensitive to transient and other power anomalies. In order to improve the power quality, the power disturbances should be monitored continuously [3]. Power quality monitoring and analysis must be able to detect and classify the disturbances on the electrical system [2]. Nevertheless, whenever the disturbance lasts for only for a few cycles, a simple observation of the waveform in a bus bar may not be enough to allow one to recognize that there is a problem or, more difficult yet, to identify the sort of the problem. One of the most important issues in power quality problems is how to detect and classify disturbance waveforms automatically in an efficient manner. Automatic disturbance recognition can be carried out with the help of wavelet transform [1]. On the other hand, the wavelet transform has been adopted in different fields, such as telecommunications and acoustics. In the last decade, the wavelet transform (WT) has been studied to analyze voltages and currents during short duration disturbances.

Wavelet analysis [1] is based on the decomposition of a signal according to time- scale, rather than frequency, using basis functions with adaptable scaling properties which are known as multi-resolution analysis. A wavelet transform expands a signal not in terms of a trigonometric polynomial but by wavelets, generated using transition (shift in time) and dilation (compression in time) of a fixed wavelet function. The wavelet function is localized in time and frequency yielding wavelet coefficients at different scales. This gives the wavelet transform much grater compact support for analysis of signals with localized transient components arising in power quality disturbances manifested in voltage, current, or frequency deviations. Several types of wavelets have been considered [2][4] for detection, and localization of power quality problems as both time and frequency information are available by multiresolution analysis. However, for classifying low-frequency and high-frequency power quality disturbances, a separate FFT routine and several neural networks are required along with the features extracted from the wavelet multiresolution analysis [5] and this procedure results in a high computational overhead. The modified wavelet transform (MWT), [6], is an extension to the ideas of wavelet transform, and is based on a moving and scalable localizing Gaussian window and has characteristics superior to either of the transforms. The modified wavelet transform is fully convertible from the time domain to two-dimensional (2-D) frequency translation domain and to then familiar Fourier frequency domain. The amplitude frequency-time spectrum and the phasefrequency-time spectrum are both useful in defining local spectral characteristics. The superior properties of the modified wavelet transform are due to the fact that the modulating sinusoids are fixed with respect to the time axis while the localizing scalable Gaussian window dilates and translates. As a result, the phase spectrum is absolute in the sense that it is always referred to the origin of the time axis, the fixed reference point.

The real and imaginary spectrum can be localized independently with a resolution in time, corresponding to the basis function in question and the changes in the absolute phase of a constituent frequency can be followed along the time axis and useful information can be extracted. The phase correction of the wavelet transform in the form of modified wavelet transform can provide significant improvement in the detection and localization of power quality disturbance transients. Using the properties of MWT and the features of the decomposed waveform, along with the AM algorithm, it is possible

\footnotetext{
* Department of Electrical and Electronics Engineering, Park College of Engineering \& Technology, Coimbatore-641659 winchandrasekar@gmail.com
} 
to extract important information from a disturbance signal and determine what type of disturbance has caused a power quality problem to occur [7]. This methodology permits on-line estimation of the system dynamic performance and may also work as a power disturbance recorder to detect, localize, and classify different disturbances by type and to store only interesting disturbances.

In this paper MWT is employed to capture the time of transient occurrence and extract frequency features of power disturbances. These MWT coefficients when applied as inputs to the neural networks require large memory space and much learning time. Hence along with MRA technique the statistical methods are used to extract the disturbance features of the distorted signal at different resolution levels. BAM is used to classify disturbance type. The learning efficiency of BAM is very fast and it is suitable for signal classification problems. Distorted signals were generated by the power system block set in MATLAB. The accuracy rate is improved using wavelets along with statistical differentiation of the various power signal disturbances.

\section{MODIFIED WAVELET TRANSFORMATION}

\subsection{Mathematical formulation}

The MWT is an extension to the ideas of WT, and is based on a moving and scalable localizing Gaussian window and has characteristics superior to either of the transforms. The local spectral information of the WT, with slight modification, can be used to perform local cross spectral analysis with very good time resolution. The phase correction absolutely references the phase of the WT to the zero time point, thus assuring that the amplitude peaks are regions of stationary phase. The excellent timefrequency resolution characteristic of the MWT makes it an attractive candidate for analysis of power system disturbance signals.

It is well known that information is contained in the phase of the spectrum, as well as in the amplitude. In order to utilize the information contained in the phase of the Continuous Wavelet Transform (CWT), it is necessary to modify the phase of the mother wavelet. The CWT $W(\tau, d)$ of a function $h(t)$ is defined as

$$
W(\tau, a)=\int_{-\infty}^{\infty} h(t) \omega(t-\tau, a) \mathrm{d} t
$$

where $\omega(t, a)$ is a scaled replica of the fundamental mother wavelet; the dilation determines the width of the wavelet and this controls the resolution. The MWT is obtained by multiplying the CWT with a phase factor as

$$
M W(\tau, f)=\exp (i 2 \pi f \tau) W(\tau, a)
$$

where the mother wavelet for this particular case is defined as

$$
\omega(\tau, f)=\frac{|f|}{\sqrt{2 \pi}} \exp \frac{-t^{2} f^{2}}{2} \exp (-i 2 \pi f t) .
$$

In equation (3) just shown, the dilation factor is the inverse of the frequency. Thus, the final form of the continuous MWT is obtained as

$$
\begin{aligned}
& M W(\tau, f)= \\
& \quad \int_{-\infty}^{\infty} h(t) \frac{|f|}{\sqrt{2 \pi}} \exp \frac{-(\tau-t)^{2} f^{2}}{2} \exp (-i 2 \pi f t) \mathrm{d} t
\end{aligned}
$$

and the width of the Gaussian window $\sigma(f)=T=\frac{1}{|f|}$.

Recently wavelet analysis was proposed in the literature as a new tool for monitoring power quality problems. Wavelet transformation has ability to analysis different power quality problems simultaneously in both time and frequency domains.

The wavelet transform is useful in detecting and extracting disturbance features of various types of electric power quality disturbances because it is sensitive to signal irregularities but insensitive to the regular-like signal behavior. Wavelet analysis deals with expansion of functions in terms of a set of basis functions, like Fourier analysis. However, wavelet analysis expands functions not in terms of trigonometric polynomials but in terms of wavelets, which are generated in the form of translations and dilations of a fixed function called the mother wavelet. Compared with Fourier transform, wavelet can obtain both time and frequency information of signal, while only frequency information can be obtained by Fourier transform.

In Fourier analysis, a signal is the series of infinite sine waves in which frequency is multiple of fundamental one. While wavelet analysis, a signal is broken in to finite approximations and details thorough wavelet function $\varphi$ and scaling function $\Phi$ will generate the approximated version. The approximations contain low frequency, and details contain high frequency. Analysis in forward direction with down sampling, we receive approximated packets, detailed packets, and wavelet coefficients. Then we up sample and include with wavelet coefficients to reconstruct the near original signal.

\subsection{Discrete MWT}

The power system disturbance signal $h(t)$ can be expressed in a discrete form as $h(k T), k=0,1,2, \ldots,(N-$ 1 ) and $T$ is the sampling interval.

The discrete Fourier transform of $h(k T)$ is obtained as

$$
H\left[\frac{n}{N T}\right]=\frac{1}{N} \sum_{k=0}^{N-1} h(k T) \exp \frac{-i 2 \pi k}{N}
$$

where $n=0,1, \ldots, N-1$.

Using equation (5), the MWT of discrete time series $h(k T)$ is obtained by letting $f \rightarrow n / N T$ and $\tau \rightarrow j T$ as

$$
M(j T, 0)=\frac{1}{N} \sum_{m=0}^{N-1} h\left(\frac{m}{N T}\right)
$$




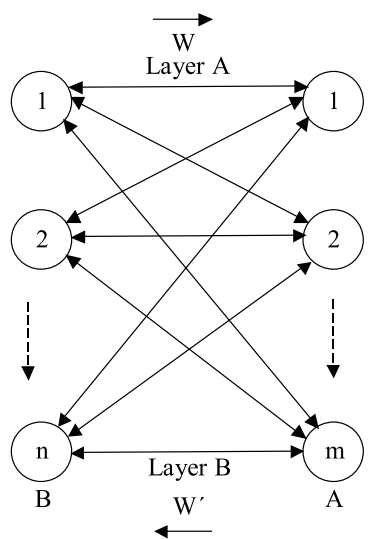

Fig. 1. BAM simplified diagram

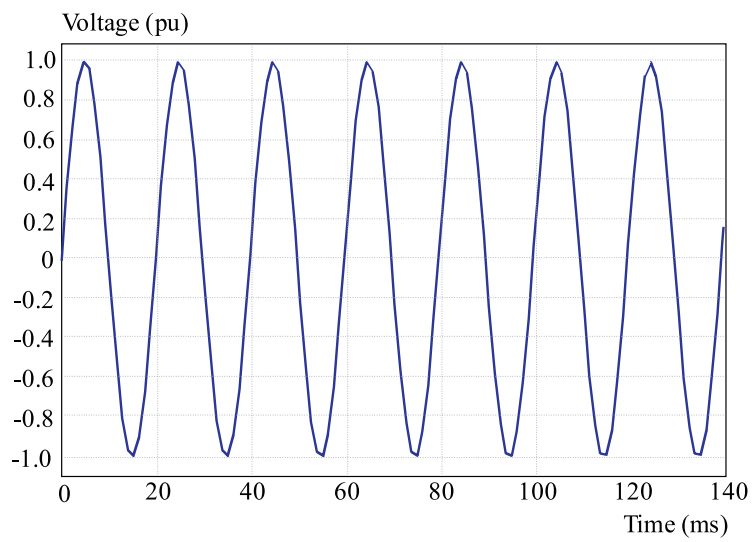

Fig. 2. Sinusoidal signal without disturbance

and $G(m, n)=\exp \frac{2 \pi^{2} m^{2} \alpha^{2}}{n^{2}}, n \neq 0$ where $j, m=$ $0,1, \ldots, N-1$ and $n=1,2, \ldots, N-1$. For $n=0$

$$
M(j T, 0)=\frac{1}{N} \sum_{m=0}^{N-1} h\left(\frac{m}{N T}\right) .
$$

Equation (7) gives the constant average of the time series into zero frequency voice, so that the averaging of the amplitude of the $M$-matrix over the time results in Fourier spectrum. Amplitude and phase of the matrix are obtained as

$$
\begin{aligned}
& |M[j T, n / N T]| \text { and } \\
& \quad\{\operatorname{IMAGE}(M[j T, n / N T]) / \operatorname{REAL}(M[j T, n / N T])\}
\end{aligned}
$$

respectively.

It can be noted that the MWT improves the WT in that it has a better resolution in phase space (i.e., a more narrow time window for higher frequencies) giving a fundamentally more sound time frequency representation. The computation of the multiresolution MWT is very efficient using convolution theorem and FFT. The total number of operations for computing MWT is $N(N+$ $N \log N)$. The frequency-time contours having the same amplitude spectrum are obtained to detect, and classify power disturbance events.

\section{MEMORY ARCHITECTURE OF BIDIRECTIONAL ASSOCIATIVE MEMORY}

Associate Memory (AM) belongs to a class of neural networks that train itself according to a certain recording algorithm. They usually acquire prior information and their connectivity (weight) matrices most often need to be formed in advance. Bidirectional Associate Memory (BAM) is a hetero associative, content addressable memory consisting of two layers. It uses the forward and backward information flow to produce an associative search for stored stimulus response association. The vector stored in the memory associate with pairs $\{a, b\}$ is given in equation (8)

$$
\left\{\left(a^{(1)}, b^{(1)}\right),\left(a^{(2)}, b^{(2)}\right), \ldots,\left(a^{(p)}, b^{(p)}\right)\right\} .
$$

It is assumed that an initializing vector $b$ is applied at the input to the layer $A$ of neurons then the neurons are assumed to be bipolar binary. The input is processed through the linear connection layer and then through the bipolar threshold functions as follows.

$$
a=\xi[W b]
$$

where $\xi[\cdot]$ is a nonlinear operator. This pass consists of matrix multiplication and a bipolar thresholding operation so that the $i$ th output is

$$
a_{i}=\operatorname{sgn}\left(\sum_{j=1}^{m} w_{i j} b_{j}\right), \text { for } i=1,2, \ldots, n .
$$

Assume that the thresholding is synchronous and the vector $a$ now feeds the layer $B$ of neurons. It is now processed in layer $B$ through similar matrix multiplication and bipolar thresholding but the processing now uses the transposed matrix $W^{t}$ of the layer $B$

$$
b=\xi\left[W^{t} a\right]
$$

or for the jth output

$$
b_{j}=\operatorname{sgn}\left(\sum_{i=1}^{n} w_{i j} a_{i}\right), \text { for } j=1,2, \ldots, m .
$$

From now on the sequence of retrieval repeated as in equation (8) or (9) to compute $a$, then as in equation (10) or (11) to compute $b$, etc. The process continues until further updates of $a$ and $b$ stop. It can be seen that in terms of recursive update mechanism, the retrieval consists of the following steps.

$$
\begin{array}{lll}
\text { First Forward Pass } & : & a^{1}=\xi\left[W b^{0}\right] \\
\text { First Backward Pass } & : & a^{2}=\xi\left[W^{t} a^{t}\right] \\
\text { Second Forward Pass } & : & a^{3}=\xi\left[W b^{2}\right] \\
k / 2 \text { th Backward Pass } & : & b^{k}=\xi\left[W^{t}, a^{k-1}\right] .
\end{array}
$$

Ideally, this back-and-forth flow of updated data quickly equilibrates usually in one of the fixed pairs $\left(a^{(i)}, b^{(i)}\right)$. 


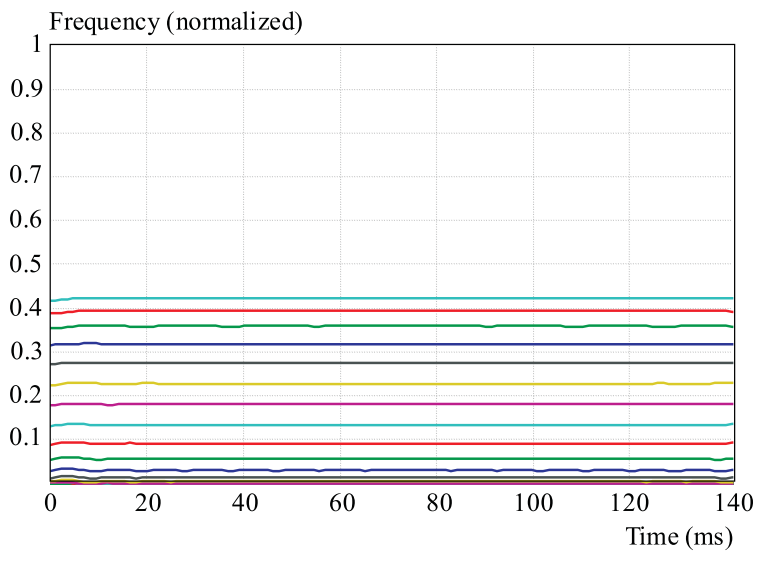

Fig. 3. MWT contour graph for pure sine signal

Figure 1 shows the simplified diagram of the BAM often encountered in the literature. Layers $A$ and $B$ operate in an alternate fashion-first transferring the neurons output signals toward the right by using matrix $W$, and then toward the left by using matrix $W^{t}$ respectively.

\section{SIMULATION \& RESULTS}

The multiresolution MWT output is a complex matrix, the rows of which are the frequencies and the columns are the time values. Each column thus represents the local spectrum for that point in time. Also, frequency-time contours having the same amplitude spectrum are obtained to detect, and classify power disturbance events. The various $\mathrm{PQ}$ disturbance signals considered for the MWT analysis are voltage sag, swell, interruption and oscillatory transients. To demonstrate the suitability of the proposed method a classification experiment is conducted IEEE 6 bus system. The disturbance is created at different location and the disturbances are recorded. The simulation is done in power system toolbox. To illustrate the use of multiresolution MWT for non-stationary signal analysis, PQ disturbance is created in a pure sinusoidal voltage waveform with data window of eight cycles. The time-frequency contours are shown in the following section.

\subsection{Sinusoidal signal without disturbances}

The pure sine wave input signal is shown in Fig. 2 and the corresponding contour of the MWT is shown in Fig. 3. Since the input is pure sine wave there is no significant change in the contour and also no disturbance has been found.

\subsection{Voltage swell}

When the normal voltage signal increases by 10 to $90 \%$, it is known as voltage swell which is shown in Fig. 4 for five cycle duration voltage swell in a pure sinusoidal voltage waveform. Figure 5 shows the time-frequency plot of the MWT contours. The pattern produces a swell in the magnitude during the disturbance and this can be directly used for classification. The time localization of the disturbance can also be seen from these contours.

\subsection{Voltage sag}

The voltage sag waveform is shown in Fig. 6 in which voltage drops about one cycle due to sudden load at node 7 . The MWT is employed to obtain the disturbance. Figure 7 shows the time frequency plot of the MWT contours for a $50 \%$ of sag in the voltage signal. From the Fig. 7 it is found that the MWT contours show a magnitude reduction during the disturbance similar to voltage sag clearly localizing and detecting the disturbance.

\subsection{High frequency disturbance}

This type of disturbance is also called oscillatory transients which are occurred due to arc fault. The time duration for this type fault is less than sag or swell type of disturbances. Figure 8 shows the disturbed signal due to switching operation. The result of MWT is shown in Fig. 9 which clearly indicates the occurrence of disturbance.

\section{CLASSIFICATION OF POWER QUALITY EVENTS}

The energy coefficients are obtained at each level. The relation between the number of decomposition level and energy coefficients is shown in Fig. 10 for different test signals. This energy curve magnifies the deviations of the signal with disturbance from the corresponding pure sinusoidal one. By using energy curve the fault can be easily detected which is applicable for all type of faults in power signals including high impedance transients. Figure 10 shows the energy coefficients of four signals on the same coordinate axis orderly.

First, 45 examples (half of all training examples) and then 90 training examples have been considered to train the ANN model. The experimental results shows, with 45 training examples, the classified accuracy rate of the distorted signals of the proposed approach is $83 \%$. When the training examples were 90 , the classified accuracy rate is $95 \%$. These results show that as the number of training example increase so does the accuracy rate. Because of the BAM model requires little learning time, the proposed approach is suitable for real time processing in a modern digital recorder.

However, for classifying low and high frequency $\mathrm{PQ}$ disturbances with noise, a separate FFT routine and several neural networks are required along with the features extracted from the wavelet analysis and this procedure results in a high computational overhead. Hence, a modified approach is required to overcome these difficulties. 


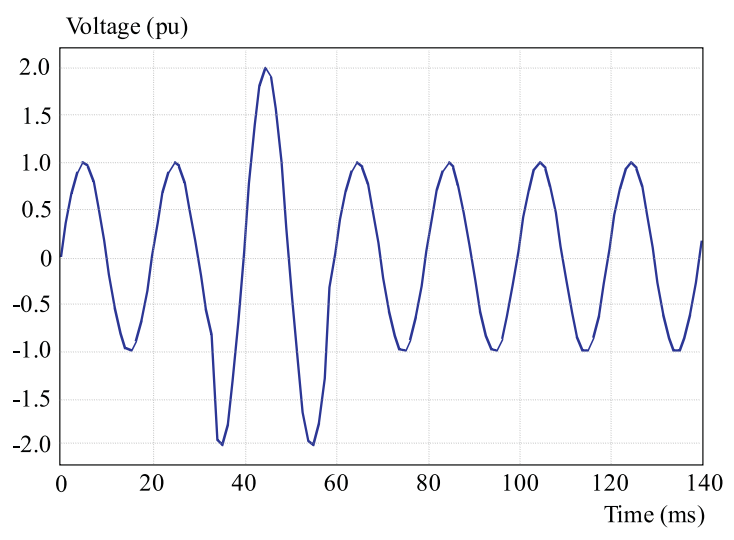

Fig. 4. Signal with swell

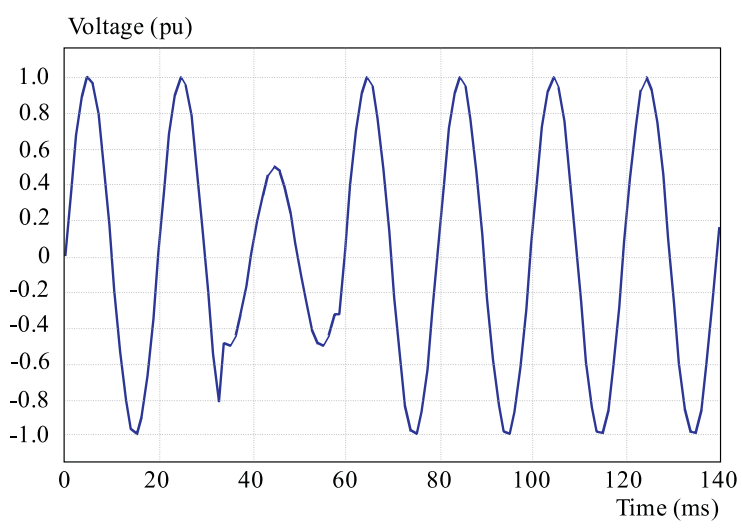

Fig. 6. Signal with sag

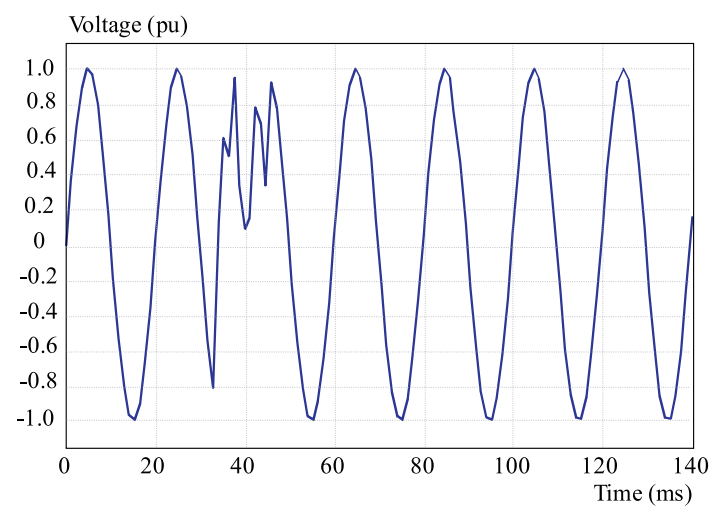

Fig. 8. Oscillatory disturbance signal

In this section, an attempt has been made to classify the three basic PQ signals (ie, voltage sag, swell, and momentary interruption). As the MWT provides us a TimeFrequency Representation (TFR) of the signal with frequency dependent resolution, the energy deviation of the TFR curve is taken as a measure to classify the signals. It is observed that during the test for different percentage of sag or swell, there is a proportionate decrease or increase in the energy deviation. The percentage decrease and increase energy deviation is given in Tables 1 and 2 respectively. It is observed that for a pure sinusoid, the energy deviation curve is linear over the entire range (this value is taken as a reference), where for the sags, the standard deviation falls below this reference value and rises

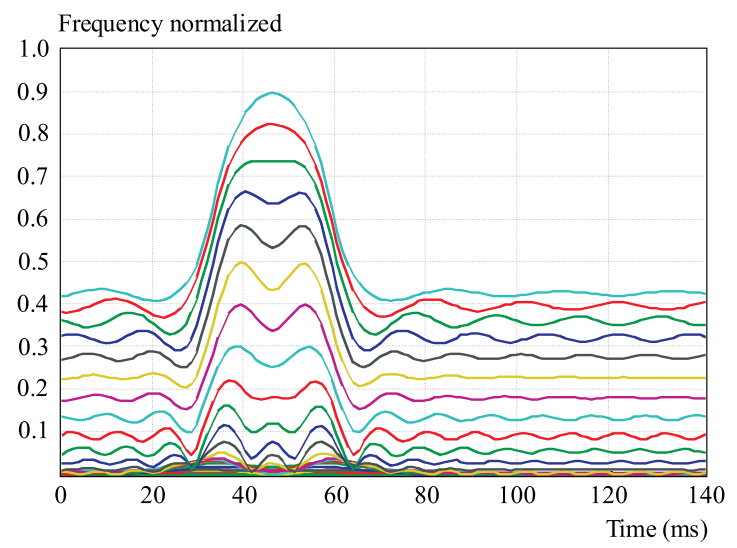

Fig. 5. MWT contour graph for a swell

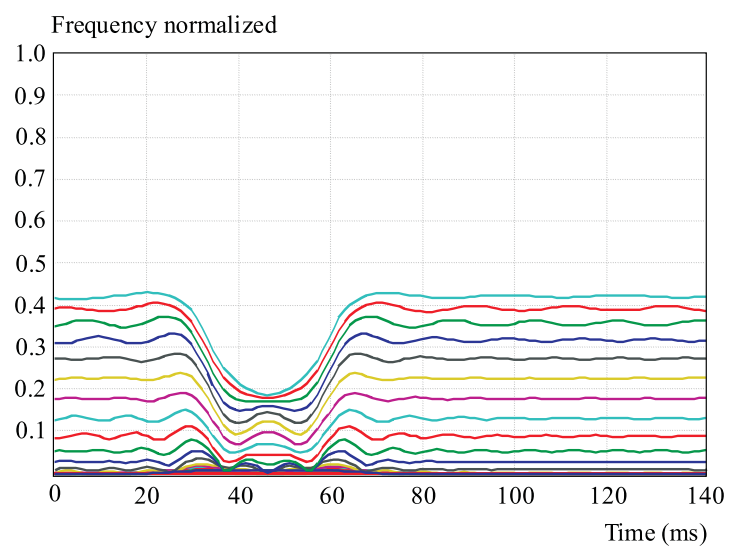

Fig. 7. MWT contour graph for a sag signal

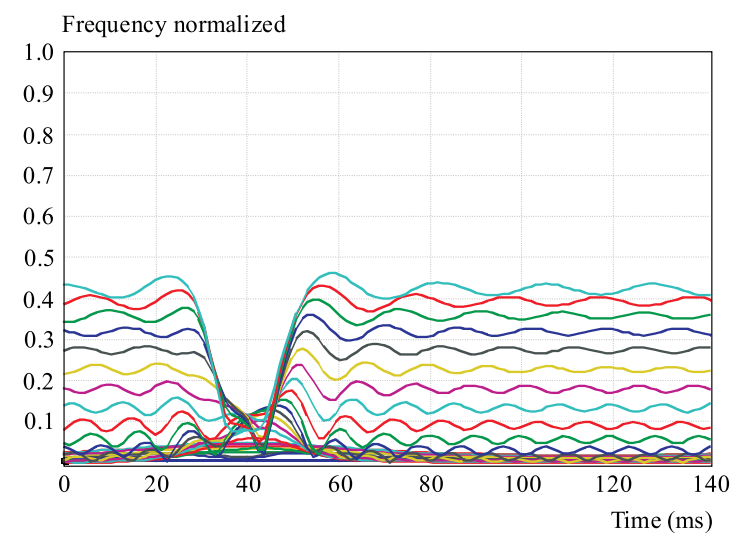

Fig. 9. MWT contour graph for an oscillatory disturbance

above the reference for the swells. The energy deviation above this is a swell and the standard deviation below it is sag. Further, it is to be noted that the FFT used in the MWT calculation provides both the amplitude and frequency components of the signal.

For classifying both steady-state and transient disturbances, energy deviation at two different Gaussian window widths $(\sigma(f)=k /|f|, k=1$ and 3) are taken and an amplitude factor is determined from the MWT matrix. These features can be used in a rule base for BAM to provide classification of the PQ events. The timefrequency plot of the MWT has a significant potential in comparison to the multiresolution analysis and energy deviation techniques is used to classify the $\mathrm{PQ}$ disturbances. 


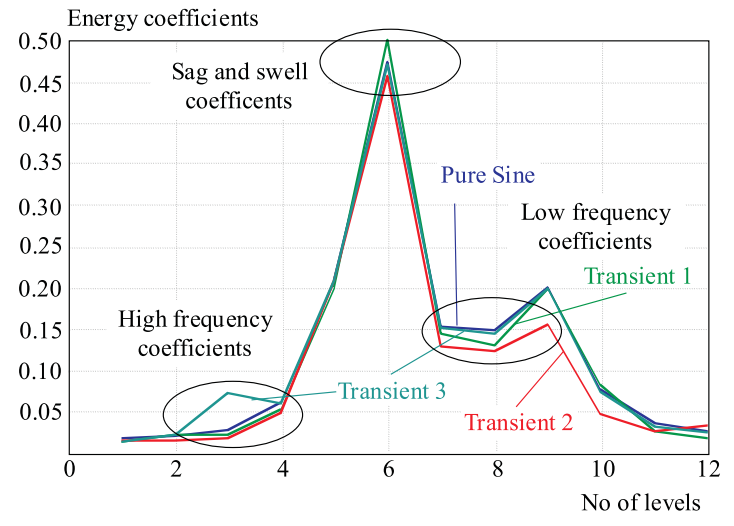

Fig. 10. Energy coefficients of different signals

Table 1. Percentage decrease in energy deviation for sag signal

\begin{tabular}{ccc}
\hline$\S$. No & \% of sag & \% decrease in energy deviation \\
\hline 1 & 10 & 12 \\
2 & 20 & 22 \\
3 & 30 & 37 \\
4 & 40 & 48 \\
5 & 50 & 60 \\
\hline
\end{tabular}

Table 2. Percentage increase in energy deviation for swell signal

\begin{tabular}{ccc}
\hline$\S$. No & \% of swell & \% increase in energy deviation \\
\hline 1 & 10 & 15 \\
2 & 20 & 26 \\
3 & 30 & 40 \\
4 & 40 & 55 \\
5 & 50 & 67 \\
\hline
\end{tabular}

Table 3. Classification test results of BAM using MWT method

\begin{tabular}{lcc}
\hline Number of training examples & 35 & 70 \\
Number of testing examples & 10 & 10 \\
Learning time (sec) & 0.01 & 0.02 \\
Recall time & 0.11 & 0.20 \\
Accuracy rate & $87 \%$ & $95 \%$ \\
\hline
\end{tabular}

Several PQ problems have been analyzed and the MWT provides an interesting and significant tool in detecting and classifying the problem. These MWT coefficients are used as input to the ANN which classify the disturbance.

\section{CONCLUSION}

This paper presents a new approach for detecting and classifying the power system disturbance in transmission lines using WP and MWT combined with BAM. In first case WP is used to detect and classify the disturbances which give better detection and classification for low frequency disturbance.

Then, MWT is proposed for both low and high frequency disturbances at different noise levels which provides better detection and classification than WP. The proposed methods are powerful tool for detection and classification of power PQ in all conditions and all types of transmission lines. The proposed approach is relatively simple, reliable and suitable for online applications.

\section{REFERENCES}

[1] DUGAN, R. C.-McGRANAGHAN, M. F.-BEATY, H. W.: Electrical Power Systems Quality, McGraw-Hill, 1996.

[2] GaOUdA, A. M.-SAlAmA, M. M. A.-SUltan, M. R.CHIKHANI, A. Y.: Power Quality Detection and Classification Using Wavelet-Multiresolution Signal Decomposition, IEEE Transactions on PowerDelivery 14 No. 4 (Oct 1999), 1469-1476.

[3] GAOUdA, A. M.-SAlAmA, M. M. A.-SUlTAN, M. R.CHIKHANI, A. Y.: Application of Multiresolution Signal Decomposition For Monitoring Short-Duration Variations in Distribution Systems, IEEE Transactions on Power Delivery 15 No. 2 (Oct 2000), 478-485.

[4] HUANG, S. J.-HSIEH, C. T.-HUANG, C. L. : Application of Wavelets to Classify Power System Disturbances, Electric Power System Research 47 No. 2 (Oct 1998), 87-93.

[5] HUANG, S. J.-HSIEH, C. T.-HUANG, C. L. : Application of Morlet Wavelets to Supervise Power System Disturbance, IEEE Transactions on Power Delivery 14 No. 1 (Jan 1999), 235-243.

[6] BRITO, N. S. D.-SOUZA, B. A.-PIERS, F. A. C. : Daubechies Wavelets in Quality of Electrical Power, 8th International Conference on Harmonics and Quality of Power ICHQP98, October 14-16, 1988, pp. 511-515.

[7] SAntoso, S.-POWER, E. J.-HOFMAnN, P.: Power Quality Assessment via Wavelet Transform Analysis, IEEE Transactions on Power Delivery 11 No. 2 (Jan 1996), 924-930.

[8] PERUniciC, B.-MALlini, M.-WANG, Z.-LIU, Y.: Power Quality Disturbance Detection and Classification Using Wavelets and Artificial Neural Networks, 8th International Conference On Harmonics and Quality of Power Proceedings, Vol. 1, 1998, pp. 77-82.

[9] DANIELS, R. F. : Power Quality Monitoring Using Neural Networks, Proceedings of the First International Forum on Applications of Neural Networks to Power Systems, July 23-26, 1991, pp. 195-197.

[10] GaOudA, A. M.-KANOUn, S. H.-SAlAMA, M. M. A.: On-Line Disturbance Classification Using Nearest Neighbor Rule, Electric Power System Research 57 (Jan 2001), 1-8.

[11] IEEE Std 1159-1995, IEEE Recommended Practice for Monitoring Electric Power Quality, November 1995.

[12] DAUBECHIES, I.: Ten Lectures on Wavelets, SIAM, Philadelphia, 1992.

[13] LAURENE, F.: Fundamentals of Neural Networks Architectures, Algorithms and Applications, Prentice Hall, 1994.

Received 27 September 2009

Perumal Chandrasekar was born in Rajapalayam, Tamilnadu in 1976. He received B.Tech (1997) from Pondicherry Engineering College and M.E (2002) in Power Systems Engineering from College of Engineering, Guindy Anna University, Chennai. He is an Assistant professor in Department of Electrical Engineering at Park College of Engineering and Technology, Coimbatore. His research interests include power quality, Wavelet analysis and artificial intelligence to power system.

Vijayarajan Kamaraj is an professor at Department of Electrical Engineering, SriSivasubramania Nadar College of Engineering, Chennai. His research interests include Electrical Machines \& Drives, power quality, and Finite element analysis. 